\title{
Organizing patient safety research to identify risks and hazards
}

\author{
J B Battles, R J Lilford
}

Qual Saf Health Care 2003;12(Suppl II):ii2-ii7

Patient safety has become an international priority with major research programmes being carried out in the USA, UK, and elsewhere. The challenge is how to organize research efforts that will produce the greatest yield in making health care safer for patients. Patient safety research initiatives can be considered in three different stages: (1) identification of the risks and hazards; (2) design, implementation, and evaluation of patient safety practices; and (3) maintaining vigilance to ensure that a safe environment continues and patient safety cultures remain in place. Clearly, different research methods and approaches are needed at each of the different stages of the continuum. A number of research approaches can be used at stage 1 to identify risks and hazards including the use of medical records and administrative record review, event reporting, direct observation, process mapping, focus groups, probabilistic risk assessment, and safety culture assessment. No single method can be universally applied to identify risks and hazards in patient safety. Rather, multiple approaches using combinations of these methods should be used to increase identification of risks and hazards of health care associated injury or harm to patients.
See end of article for authors' affiliations

Correspondence to: Dr J B Battles, Agency for Healthcare Research and Quality, Center for Quality Improvement and Patient Safety, 540 Gaither Road, Rockville, MD 20850, USA; jbattles@ahrq.gov
$\mathrm{P}$ atient safety has become a major concern throughout the world. In the USA, ${ }^{1}$ the UK, ${ }^{2}$ and in a number of other countries, major patient safety research efforts have been launched. Two significant questions challenge the patient safety effort: (1) How can we organize a comprehensive patient safety research initiative? (2) What are the appropriate research methods to be used for carrying out the research initiative? Eisenberg's $\mathrm{s}^{3}$ analogy of patient safety having the characteristics of an epidemic of worldwide portions may provide some guidance to answering the first question. He outlined a three stage research continuum using the epidemic metaphor as an organizing principle for a patient safety initiative.

- Identify the risks and hazards that cause or have the potential to cause health care associated injury or harm.

- Design, implement, and evaluate patient safety practices that eliminate known hazards, reduce the risk of injury to patients, and create a positive safety culture.
- Maintain vigilance to ensure that a safe environment continues and patient safety cultures remain in place.

Clearly, different research methods and approaches are needed at each of the different stages of the continuum. There is a good deal of concern that the research approaches that have been associated with health services research and the study of medical outcomes may be inadequate to meet the needs and problems associated with patient safety. The methods that are appropriate for identifying risks and hazards are quite different from those for designing interventions, while the research methods for documenting the adoption and use of safe practices are different still.

Patient safety includes topics such as the use of information technology to reduce error which have a long and rich tradition in health care, and others such as simulation and human factors engineering which have only recently started to have a significant impact in the clinical domain. While many aspects of the study of patient safety are relatively new, they should build on the rich heritage of safety science that has existed outside medicine for years. There is no single research method that can be universally applied to patient safety. Rather, we must look to multiple approaches to deal with the various stages of the patient safety research action plan. Is it possible to build upon traditional approaches from health services research and add the strengths of research approaches from safety science and other disciplines to learn lessons for patient safety research?

This paper will outline a framework for approaching patient safety research and discuss various research methods that could be applied to conducting research in the first stage of this new and emerging field of patient safety.

\section{A CONCEPTUAL FRAMEWORK FOR PATIENT SAFETY RESEARCH}

As with any new field or discipline, there are goals that should guide the focus of research and key terms that influence the conceptual framework that ultimately shape the research approaches to meet the ultimate goals. So it is with patient safety.

\section{Key terms}

In this paper we use the phrase health care associated injuries to describe patient harm or injuries. This term implies patient safety events which cause or have the potential to cause harm. Health care associated injuries are those associated with the process or structure of care rather than with a patient's underlying or physiological, 


\section{Box 1 Definitions of patient safety event types}

- Adverse/harm events are occurrences during clinical care that result in physical or psychological injury or harm to a patient or harm to the mission of the organization.

- No harm events are events that have occurred but result in no actual harm although the potential for harm may have been present. Lack of harm may be due to the robust nature of human physiology or pure luck. An example of such a no harm event would be the issuing of an $A B O$ incompatible unit of blood for a patient, but the unit was not transfused and was returned to the blood bank.

- Near misses are defined as events in which the unwanted consequences were prevented because there was a recovery by identification and correction of the failure, either planned or unplanned. ${ }^{6}$

- Dangerous situations are where both human and latent failure exists that creates a hazard increasing the risk of harm. Information may be collected from individuals familiar with the process of care in organizations about conditions that are highly likely to cause an injury to a patient or patients.

environmental, or disease related antecedent conditions. We have also chosen to focus on association rather than causation. Risk is the possibility/probability of occurrence or recurrence of an event multiplied by the severity of the event. ${ }^{2}$ Hazard is anything that can cause harm. ${ }^{2}$ Both risks and hazards reveal themselves nested within an event. An event has been described as deviations in activities or technologies which lead towards unwanted negative consequences. ${ }^{4}$ In the case of patient safety, the negative consequences are health care associated injuries/harm or the potential to cause such injuries/harm. Battles and Shea ${ }^{5}$ have classified the event types into different categories (box l).

\section{Goal}

The goal of patient safety is to reduce the risk of injury or harm to patients from the structure and process of care (box 2 ). This can be accomplished by eliminating or minimising unintended risks and hazards associated with the structure and process of care.

Given this focus on health care associated injury contained within patient safety events, we can have a vision for patient safety as "zero health care associated injuries or harm".

\section{Structure and process}

Clearly, patient safety research needs to focus on the sources of risks and hazards that can lead to health care associated injury. The process-structure-outcome model described by Donabedian $^{7}$ is a helpful concept for describing the focus of health services research. The aim of safety research must be to improve outcome- that is, to minimize harm to patients through failures in the process or structure of care. Improvements in safety will come from changes (improvements) in these areas. Research is needed to determine the relative safety of processes and structures. Of course, not all adverse outcomes (including death) are due to problems with either the process or structure of care. In reality, the process of care occurs within the structure of the healthcare system. One criticism of examining only the outcomes of care is that it does not take into account the patient's condition before

\section{Box 2 Goal and vision for patient safety}

- The goal of patient safety is to reduce the risk of injury or harm to patients from the structure and process of care. This can be accomplished by eliminating or minimising unintended risks and hazards associated with the structure and process of care.

- A vision for patient safety would be "zero health care associated injuries or harm".

entering the healthcare system. Coyle and Battles ${ }^{8}$ modified the Donabedian approach, emphasising the need to account for a patient's antecedent conditions. Some patients enter the healthcare system with antecedent conditions that are incompatible with life. Nothing that is done for these patients changes an adverse outcome-death or serious disability. While health services must do all they can to mitigate the effects of antecedent events over which they have no control, it is important to bear in mind that attempts to "control" for such differences statistically are fallible. This is because a statistical adjustment for case mix - that is, for antecedent conditions-is an uncertain science. This has obvious implications for those who seek to use outcomes in a system of sanction or reward to grade institutions or even to determine which institutions require further examination.

Although the antecedent condition confounds comparisons between institutions, complex patients with co-morbid conditions do test the resilience of the system. Thus, when it comes to the investigation of particular events, careful examination of what went wrong in a complex case may identify weakness in a system which could put healthier patients at increased risk. In this sense, such complex cases are like the canary in the mine shaft. For example, administration of an incompatible unit of blood in an emergency might lead to the identification of weak systems which could eventually place a patient requiring elective transfusion at risk. Such weakness could lie either in the structure or the process of care. Figure 1 is an illustration of the patient safety version of the Donabedian model showing structure within process. Rather than the usual boxes aligned in linear fashion, the illustration presents the process as occurring within the structure of health care.

\section{Types of risks and hazards}

What are the sources of these risks and hazards that are embedded in the process and structure of care? Reason ${ }^{9}{ }^{10}$ has classified two categories of failure (risks and hazards) based on who initiated them and how long it takes to have an adverse effect. Active failures are those committed by

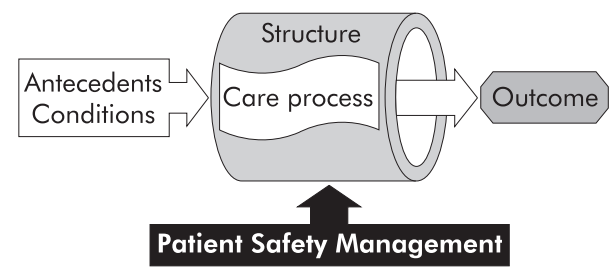

Adjust structure and process to eliminate or minimize risks of health care associated injury before they have an adverse event that impacts on the outcomes of care

Figure 1 A structure and process model for patient safety based on Donabedian. 
individuals in direct contact with the human system interface. These active failures are often referred to as human error. Errors can be considered actions or inactions that lead to deviations from intentions or expectations. Errors are most often manifested as risks that either lead to or have potential to lead to harm. Latent failures are created by the delayed consequences of technical and organizational actions and decisions. Latent failures or conditions are also sometimes referred to as system errors; however, they might more accurately be considered as hazards embedded within the structure and process of the healthcare system rather than risks associated with human behavior.

Rasmussen ${ }^{11}$ looked at the cognitive basis for human behavior underlying failures or error and identified three types—skill based, rule based, or knowledge based-which, again, are considered specific types of risk. Knowledge based behavior involves the conscious application of existing knowledge to manage novel situations. Rule based decision making involves the application of existing rules or schemes to manage familiar situations. Prolonged active processing is not required; simply the selection and application of the appropriate rule. Mistakes are rule based failures of planned actions/rules to be completed as intended or selecting the wrong rule to achieve an aim. ${ }^{9}{ }^{10}$ Skill based behaviors refer to "automatic" tasks requiring little or no conscious attention during execution. Slips are inadvertent skill based failures of commission (doing the wrong thing). ${ }^{9}{ }^{10}$ While lapses are skill based failures of knowing what to do but failing to do it, omitting a step, or losing one's place in a process-failures of omission. ${ }^{10}$ Fumbles are skill based failure of whole body movement such as dropping something. ${ }^{10}$ A glossary of patient safety terms is shown in box 3 .

Van der Schaaf ${ }^{6}$ has organized the active failures and latent conditions into human, organizational, and technical failures. Relating this classification schema to the Donabedian model, we would have process within structure and human behavior nested within process. Active or human failures represent risks to process, with latent conditions representing hazards embedded in the process and structure (organizational or technical) of health care. As Reason ${ }^{9}{ }^{10}$ has pointed out, it is a combination of active failures interacting with latent conditions that result in adverse events. Figure 2 is a representation of this nested concept within the framework of the Donabedian model.

Human failures or error are nested within latent conditions or hazards that make up the continuum of events. Adverse/ harm events are those where actual harm and/or injury has taken place with the harm representing some degree of severity from death to minor injury. There are, of course, events that occur that involve human failure and an interaction with latent conditions, but the outcome does not result in actual harm to the patient. These "no harm" events represent potential rather than actual harm with warning levels of potential severity as well. The "near miss" event, on the other hand, does not manifest itself in actual harm to a patient because there was intervention and recovery. Again, there is potential for harm with a similar level of potential severity.

Detection or identification is the first step in error (patient safety) management. ${ }^{12}$ From an organizational point of view it is important that detection or identification rates are high because unintended risks and hazards that are not detected can have disastrous consequences. For the overall detection process to be effective it needs to identify not only adverse/ harm events, but no harm and near miss events as well. Adverse events caused by unintended risks and hazards are much less common than no harm and near miss events. Rather than wait for the adverse event, it is essential to detect and correct the unintended risks and hazards.

\section{Box 3 Glossary of patient safety terms}

- Health care associated injuries are those injuries associated with the process of care rather than with a patient's underlying or physiological, environmental, or disease related antecedent conditions.

- Errors are actions or inactions that lead to deviations from intentions or expectations. Errors can include problems in practice, products, procedures, and systems.

- Events are deviations in activities or technologies which lead towards unwanted negative consequences. ${ }^{4}$

- Risk is the possibility/probability of occurrence or recurrence of an event multiplied by the severity of the event. $^{2}$

- Hazard is anything that can cause harm. ${ }^{2}$

- Active failures are errors and violations committed by those in direct contact with the human-system interface. ${ }^{9} 10$

- Sharp end individuals are in direct contact with the human-system interface. In health care, sharp end individuals administer care to patients. Their actions and decisions may result in active failures. ${ }^{9} 10$

- Latent conditions are hazards resulting from the delayed consequences of technical and organizational actions and decisions. These underlying conditions may predispose sharp end individuals to fail. 910

- Blunt end individuals take actions and/or make decisions that affect technical and organization policy and procedures and allocate resources. Their actions and decisions may result in latent conditions. Examples of such decisions include those related to staffing and resource allocation. ${ }^{78}$

- Knowledge based behavior involves the conscious application of existing knowledge to manage novel situations. ${ }^{11}$

- Rule based behavior is decision making involving the application of existing rules or schemas to manage familiar situations. ${ }^{11}$

- Skill based behavior refers to automatic tasks requiring limited or no cognitive attention during execution. ${ }^{11}$

- Slips are inadvertent skill based failures of commission (doing the wrong thing). ${ }^{9} 10$

- Lapses are skill based failures of knowing what to do but failing to do it, omitting a step, or losing one's place in a process - failures of omission. ${ }^{10}$

- Fumbles are skill based failure of whole body movement such as dropping something. ${ }^{10}$

- Mistakes are rule based failures of planned actions/ rules to be completed as intended or selecting the wrong rule to achieve an aim. ${ }^{910}$

- Safe practices are those proved to reduce the risk of adverse events related to exposure to medical care across a range of diagnoses or conditions. ${ }^{22}$

Thus, one of the goals of patient safety management is to increase the detection and reporting rates of patient safety events in order to decrease risk of harm to patients. The detection sensitivity level (DSL) is the number of patient safety events containing actual or potential unintended risks and hazards identified by an organization. For a given true 


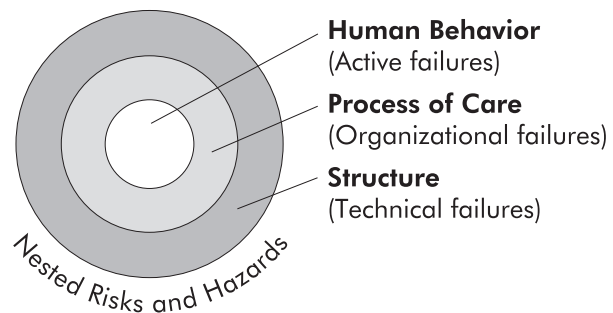

Figure 2 A model for the nested relationship of risks and hazard in patient safety.

number of hazards, high reporting rates occur when the DSL is high, while few events reported is indicative of a low DSL. To achieve a high DSL an organization must eliminate impediments to reporting. The event severity level (ESL) is the level severity (actual or potential) for each of the events reported. Over time the ESL, both actual and potential, should go down as an indicator of successful patient safety management while the ratio of events reported to actual harm remains high. ${ }^{13}$ Figure 3 is an illustration of this concept of DSL and ESL.

\section{THE RESEARCH METHODS CONTINUUM}

Given the conceptual framework for patient safety research, what methods would fit the continuum? Not surprisingly, most patient safety research to date has been devoted to stage 1 of the epidemic cycle with a focus on identification of risks and hazards to patients from health care associated injury or harm. It is for this reason that we devote most of our discussion of methods to stage l-identifying risks and hazards.

\section{STAGE 1 RESEARCH METHODS}

Thomas and Petersen ${ }^{14}$ have identified a continuum of research methods for stage 1 . This continuum flows from latent or organizational risks and hazards to more active failures leading to injury or harm. Their continuum moves from incident reporting to chart review to direct observation. At stage 1 of the epidemic cycle research methods must be judged on how well they are able to identify risks and hazards within the human behavior nested within process within structure. At later stages, interventions to reduce error and harm should be evaluated and this requires an unbiased method for measurement of these outcomes.

\section{Use of archival records}

Several types of archival records are frequently used as a source of patient safety related error and harm, including the use of medical charts, records, chart abstracts, electronic heath records (EHR) for surveillance of indicators of harm, and administrative data based on discharge or billing records.

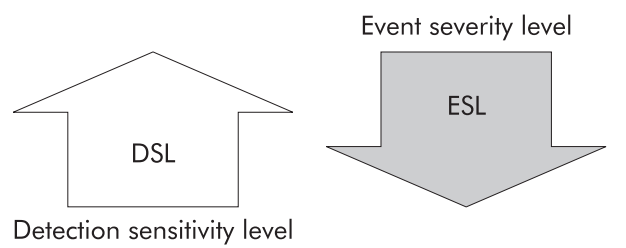

Figure 3 Relationship between detection sensitivity level (DSL) and event severity level (ESL).
The classic studies of medical error cited in "To Error is Human" ${ }^{\prime 1}{ }^{15}{ }^{16}$ used the medical chart or record review method as well as studies conducted in other countries. ${ }^{17}$ This approach is an excellent method for identifying indicators of harm as documented in the record. However, record reviews can be expensive to conduct and are time consuming. Determining rate based information on unintended hazards within the process of care based on case notes and chart reviews can be problematic because adverse event rates are biased, even after allowance has been made for case mix. We propose, instead, a method based on opportunity for error rather than the patient as the denominator. Another form of chart review is the use of chart abstraction to identify health care associated injuries and harm. This method is promising if the abstraction system is already in place. Large scale use of randomly selected charts from known populations such as the Medicare population in the US can provide accurate estimates of the rate of occurrence of known injuries or harm. Another new and promising method for identification is the use of triggers or indicators of harm designed to operate within electronic or paper record systems. Like the use of abstraction systems, such surveillance methods look for known indications of harm that may have or are about to occur. Such a surveillance system can be run in the background and have a high yield for capturing events that would otherwise go unnoticed and unreported. While the use of record based identification works well for known harms, it does less well for spotting rare or unusual events. If the information is not in the chart or in the EHR system, it cannot be used. Archival records cannot be used effectively to identify root causes and contributing factors, nor for some no harm events and all near misses.

Administrative data based on discharge or billing information represent a large set of data that can be used to detect indicators of patient safety problems. Many organizations have access to large administrative data sets which can be used to determine indicators of concern for patient safety and harm to patients. Recently, investigators have been exploring the use of such data to determine indicators of concern for patient safety. These patient safety indicators are a set of codes from the International Code of Diseases (ICD) 9 or 10 which provide an indication of a patient safety concern. Because the data were collected initially for other purposes, administrative data have some limitations based on the potential for coding variation and the fact that they do not directly measure health care associated harm, only indications of harm.

\section{Spontaneous active event reporting}

The use of spontaneous active event reporting systems as a means of identifying risk and hazards including harm, no harm, and near miss events has received considerable attention in both the US and the UK. Spontaneous reporting refers to the fact that a report is generated in response to an event that has occurred. It is active in the sense that someone-mostly sharp end healthcare providers-must actively report the event. The major criticism of this type of approach to the identification of risks and hazards is the fact that events are often under reported. ${ }^{18}$ Thus, from an epidemiological perspective, any estimates of rates or baseline denominators are very unreliable, leading some to suggest that such a system should be abandoned. Helmreich ${ }^{19}$ and others point out, however, that these reporting systems should not be used to determine rates. They argue that they are extremely valuable in identifying latent failures associated with no harm and near miss events that might not otherwise be detected. A further strength of these systems is the involvement of sharp end health professionals in looking for and reporting patient safety threats. These reporting 
systems are also affected by the safety culture of an organization. Changes in reporting rates may signal changes in the safety culture either in a positive or negative manner.

When an event is reported an institution may ask whether this event has been seen before and, if so, how many times. Most event reporting systems cannot identify specific event types and characteristics that are user defined. Methods using case based reasoning to identify patterns of similar cases, both at the level of a single institution and at the large central data system level, are beginning to show promise. These approaches to data retrieval or data mining do not use the familiar statistical approaches that have commonly been used in health services research, but look for closeness of fit to key features of an event.

Root cause analysis is another research approach that is most commonly associated with traditional event reporting approaches. Extensively used in a variety of industries for decades, root cause analysis provides a retrospective analysis of the factors that lie behind the consequent event. Root cause analysis cannot be used with archival records with any degree of accuracy. There are those who criticize the use of root cause analysis because they are uncontrolled case studies and it is often impossible to show a statistical correlation between cause and outcome. ${ }^{20}$ This criticism has led to shifting the focus of a root cause analysis from the identification of causes of an event to the identification of contributing factors. The contribution of hindsight bias is another issue that has been raised in the use of root cause analysis where knowledge of the outcome influences or biases any retrospective study. Despite this criticism, the classification of causes and their use with causal trees has been extensively validated in a number of settings.

\section{Direct observation}

The accurate identification of human failures often requires direct observation of performance. Direct observation using ethnographic approaches is an extremely powerful technique for studying a variety of social phenomena including safety issues. Watching actions unfold in a natural setting provides the observer with real time relationships to the full context in which events occur. One limitation to observation is that the observer may or may not observe a patient safety event during the period of observation. The limitations of the human observer are also limitations to direct observation approaches. The use of video taping to record actual events as they occur has proved to be a powerful tool in studying human behavior. The use of video monitoring as a method of identifying human failures and risks during the process of care is becoming more widely used in emergency rooms, intensive care units, and during surgery. Limitations of video recording are related to the limits of the audio and video equipment being used. What is observed is limited by the camera's viewing range or field of view and fidelity. Audio recording of contextual sound can be a limitation in a noisy clinical care setting. Analysis of video recording is time consuming and requires extensive coding and classification to become truly useful.

\section{Process mapping and probabilistic risk assessment}

Process mapping is a powerful technique which has been used in a number of industries to identify potential failure points and system breakdowns that represent significant risks and hazards. The application of process mapping approaches is gaining greater acceptance in a number of clinical areas as a valid approach to identifying potential hazards and risks associated with various clinical processes. Another approach that is often related to process mapping is that of probabilistic risk assessment (PRA). ${ }^{21}$ Designers of nuclear power plants, aircraft, and spacecraft have been using this technique for decades. However, many applications

\section{Key messages}

- Patient safety research initiatives can be organized in three stages:

- to identify the risks and hazards;

- to design, implement, and evaluate patient safety practices; and

- to maintain vigilance to ensure that a safe environment continues and patient safety cultures remain in place.

- The aim of safety research must be to improve outcome-that is, to minimize harm to patients through failures in the process or structure of care. A modification of Donabedian's model of process occurring within structure of care is a helpful concept for identifying the embedded risk and hazards.

- There are no perfect methods for identifying risks and hazards in patient safety. Each research method has strengths and inherent weaknesses. The use of combinations of methods using archival records, event reporting, observation, and risk assessment methods is required to identify risks and hazards in patient safety.

of PRA have been applied most often to mechanical systems rather than to work processes that have a high degree of human interaction such as exists in health care. Sociotechnical probabilistic risk assessment (ST-PRA) combines the best of rigorous and well tested engineering methodologies with the science of human factors to provide a new methodology for modelling human systems.

\section{ASSESSING SAFETY CULTURE}

Safety culture assessment is an essential element of research at stage 1 of the epidemic cycle. Without the proper safety culture in place, it is almost impossible to identify fully the risks and hazards that can lead to health care associated injury. Measuring safety culture has become an important research priority. What are the elements of safety culture and how are they most effectively measured? Assessment of organizational culture has been an important research activity within the disciplines of organization psychology and sociobiology. These fields have produced valid and reliable methods and approaches for assessing culture within the healthcare field. A major challenge to the development of a cultural assessment instrument is overcoming the natural tendency for social desirability in directing the response to critical questions that can limit the value of the data collected. Careful design and validation are essential for effective survey instrumentation and useful results.

\section{DISCUSSION}

There are no perfect methods for identifying risks and hazards in patient safety. Each research method has strengths and inherent weaknesses. The debate as to which method is best should shift to how to combine the strengths of various approaches while minimising the weakness of each. The use of combinations of methods is likely to yield a much better result in learning about risks and hazards of health care associated injury or harm. An example of this combined approach might be the use of screening for indicators of harm from administrative data followed by a more focused review of medical records or chart abstracts in the area of interest. If a critical care process is identified from either event reporting or archival record reviews, it can be followed up by combinations of direct observation, video 
observation, and/or probabilistic risk assessment and process mapping.

In the process of identifying risks and hazards we need to employ multiple methods to truly understand or fix our position as to where we are in patient safety. We should apply a principle of maritime navigation which states that you can never truly know where you are without a three point fix of your position.

\section{Authors' affiliations}

J B Battles, United States Department of Health and Human Services, Agency for Healthcare Quality and Research, Center for Quality Improvement and Patient Safety

R J Lilford, University of Birmingham, UK National Health Service, Research and Development Directorate Methodology Programme

The opinions and assertions contained herein are the private views of the authors and are not to be construed as official or as reflecting the views of the Agency for Healthcare Research and Quality, the United States Department of Health and Human Services, or the UK Department of Health.

\section{REFERENCES}

1 Kohn LT, Corrigan JM, Donaldson MS, eds. To err is human; building a safer health system. Washington, DC: National Academy Press, 1999

2 Department of Health. An organization with a memory: a report of an expert group on learning from adverse events in the NHS. London: National Health Service, 2000.

3 Eisenberg JM. Medical errors as an epidemic. A presentation at the National Summit on medical errors and patient safety research. Washington, DC: The Quality Interagency Coordination Task Force (QuIC), September 2001.

4 Freitag M, Hale A. Structure of event analysis. In: Hale A, Wilpert B, Freitag M, eds. After the event: from accident to organization learning. New York: Elsevier, 1997:11-22.

5 Battles JB, Shea CE. A system of analyzing medical errors to improve GME curricula and programs. Acad Med 2001;76:127-33.
6 Van der Schaaf TW. Near miss reporting in the chemical process industry, PhD Thesis. Eindhoven, NL: Eindhoven University of Technology, 1992.

7 Donabedian A. Explorations in quality assessment and monitoring: the definition of quality and approaches to its assessment, Volume I. Ann Arbor, Ml: Health Administration Press, 1980.

8 Coyle YM, Battles JB. Using antecedents of medical care to develop valid quality of care measures. Int J Qual Health Care 1999;1 1:5-12.

9 Reason J. Human error. New York: Cambridge University Press, 1990:175.

10 Reason J. The organizational accident. New York: Ashgate, 1997.

11 Rasmussen J. Outlines of a hybrid model of the process operator. In: Sheridan TB, Johannsen G, eds. Monitoring behavior and supervisory control. New York: Plenum Press, 1976.

12 Zapt D, Reason JT. Introduction to error handling. Appl Psychol 1994;43:427-32.

13 Kaplan HS, Battles JB, Van der Schaaf TW, et al. Identification and classification of the causes of events in transfusion medicine. Transfusion 1998;38:1071-81.

14 Thomas EJ, Petersen LA. Measuring errors and adverse events in health care. $J$ Gen Intern Med 2003; 18:61-7.

15 Brennan A, Leape LL, Laird NM, et al. Incidence of adverse events and negligence in hospitalized patients: results of the Harvard medical practice study I. N Engl J Med 1991;324:370-6.

16 Thomas EJ, Studdert DM, Burtin HR, et al. Incidence and types of adverse events and negligence care in Utah and Colorado. Med Care 2000;38:261-71.

17 Vincent C, Neale G, Woloshynoych M. Adverse events in British hospitals: preliminary retrospective record review. BMJ 2001;322:517-9.

18 Cullen D, Bates D, Small S, et al. The incident report system does not detect adverse events: a problem for quality improvement. Jt Comm J Qual Improv 1995;21:541-8.

19 Helmreich RL. On error management: lessons from aviation. BMJ 2000;320:781-5.

20 Wald W, Shojania KG. Root cause analysis. In: Shojania KG, Duncan BW, McDonald KM, Wachter RM, eds. Making healthcare safer: a critical analysis of patient safety practices, Evidence report/technology assessment number 43. Rockville, MD: Agency for Healthcare Research and Quality, 2001:51-6.

21 Mosleh A, Bari RA, eds. Probabilistic safety assessment and management. New York: Springer, 1998.

22 Shojania KG, Duncan BW, McDonald KM, Wachter RM, eds. Making healthcare safer: a critical analysis of patient safety practices, Evidence report/technology assessment number 43. Rockville, MD: Agency for Healthcare Research and Quality, 2001. 